\title{
ICARUS detector: present and future
}

\author{
F. Varanini (on behalf of the ICARUS Collaboration) \\ ${ }^{1}$ INFN Sezione di Padova, via Marzolo 8, Padova, Italy
}

\begin{abstract}
The ICARUS-T600 detector is the first large-scale liquid Argon TPC ever operated; its successful data-taking campaign at Gran Sasso laboratory, with CNGS beam and atmospheric neutrinos, proved the maturity of this innovative detection technology. In particular, it performed a search for sterile neutrinos through $v_{\mu}$ to $v_{e}$ oscillations, observing no excess w.r.t. backgrounds and allowing to strongly constrain the parameter space, indicating a narrow region in agreement with all experimental data.

A definitive answer to the sterile neutrino puzzle will be given by the SBN experiment at the Booster beam at FNAL, where ICARUS-T600 will act as far detector, by performing both appearance and disappearance measurements.
\end{abstract}

\section{The sterile neutrino puzzle}

Neutrino oscillations represent one of the greatest discoveries in recent particle physics; the vast majority of experimental results in the last 20 years appear consistent with a 3-neutrino scenario, characterized by three flavor eigenstates $\left(v_{e}, v_{\mu}, v_{\tau}\right)$ and three corresponding mass eigenstates, with small mass differences $\left(\Delta m_{12}^{2} \sim 8 \cdot 10^{-5} \mathrm{eV}^{2}, \Delta m_{23}^{2} \sim 2.5 \cdot 10^{-3} \mathrm{eV}^{2}\right)$. However, a few anomalous results pointed out to possible oscillations mediated by a much larger $\Delta m^{2}$, suggesting the existence of (at least) a fourth mass eigenstate:

- The observation of a $v_{e}$ excess in $v_{\mu}$ beams from accelerators, at experiments such as LSND $(\sim 3.8 \sigma$ effect) and MiniBoone;

- A deficit w.r.t. expectations (also $\sim 3 \sigma$ ) in the $\overline{v_{e}}$ flux from nuclear reactors;

- A similarly lower signal from high-intensity $v_{e}$ sources used in the calibration of solar neutrino experiments.

While these results are not statistically compelling, the experimental scenario concerning the possible sterile neutrinos is far from clear, and requires a full clarification.

\section{The ICARUS-T600 experiment at LNGS}

The ICARUS-T600 data-taking run at the underground LNGS laboratory, lasting from 2010 to 2013, represents the first large-scale physics experiment with a liquid Argon Time Projection Chamber ( $L A r$ $T P C$ ), with a detector active mass of $\sim 470$ tons.

The LAr-TPC technique exploits the ionization of LAr, generating electrons $(\sim 5000$ per mm, for a m.i.p.) that drift towards an anodic wire-plane array; the stereoscopic, non-destructive read-out 
of wire signals allows a 3D reconstruction of any ionizing track with a millimeter-scale resolution. Moreover, the VUV scintillation light produced in the Argon, detected by PMTs immersed in the detector, is used for signal timing and trigger generation.

The ICARUS-T600 detector $[1,2]$ was exposed to the CNGS neutrino beam from CERN, collecting a total statistics of $8.6 \cdot 10^{19}$ protons on target, with a detector live-time higher than $93 \%$. It also recorded atmospheric neutrinos with a total exposure of $0.73 \mathrm{kton} \times$ year.

The concentration of electronegative impurities (essentially $\mathrm{O}_{2}$ ) in LAr is a crucial parameter for the operation of a LAr-TPC; a large contamination would absorb a significant fraction of drift electrons, resulting in a degradation of signals. Significant experimental effort must be devoted to the Argon purification and recirculation; in the ICARUS-T600 case, the drift electron lifetime was kept higher than $7 \mathrm{~ms}$ during the whole data-taking period, as shown in figure 1; this results, obtained through continuous Argon recirculation both in the liquid and gas state, ensures a small signal attenuation during the drift (12\% for a drift length of 1.5 meters) and represents an important milestone for future LAr-TPC with much larger drift lengths [3].

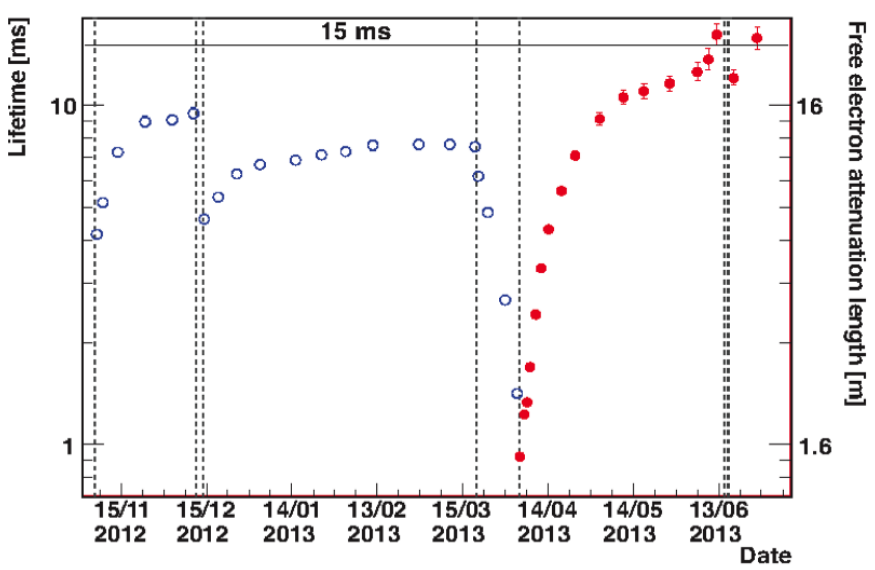

Figure 1. Electron lifetime as a function of time, during the last 7 months of the ICARUS-T600 run at LNGS. Dashed lines indicate periods were recirculation was not active, while the increase in the final weeks is due to the installation of a new, more powerful recirculation pump.

\subsection{Search for atmospheric neutrinos}

The analysis of the sample of atmospheric neutrinos recorded by ICARUS-T600 at LNGS $(\sim 0.73 \mathrm{kt})$ is ongoing; about $25 \%$ of the collected statistics has been analyzed so far. The search concentrated on multi-prong interaction vertex topologies with at least two charged particles, corresponding to $\sim 30 \%$ of $v_{\mu} \mathrm{CC}$ events.

An automatic filter algorithm allows identifying this kind of topologies, reducing the amount of events to be visually scanned by a factor $\sim 200$; in the analyzed statistics, 12 atmospheric neutrino interactions ( 2 electron-like, 3 muon-like) have been identified, in agreement with the expectation of $10 \pm 2$ events. 


\subsection{Measurement of muon momentum via multiple Coulomb scattering}

Multiple Coulomb scattering $(M C S)$ is the only way to compute the momentum of non-contained muons, such as the ones from $v_{\mu}$ charged current interactions. A dedicated algorithm developed for ICARUS-T600 was tested on a sample of 1000 stopping muons, i.e. muons from CNGS neutrino interactions in the upstream rock, entering the active TPC volume and stopping or decaying there. This sample is an ideal benchmark for the MCS measurement, providing an independent calorimetric estimate of momentum, in an energy range $(1 \div 5 \mathrm{GeV} / \mathrm{c})$ relevant for future neutrino experiments.

The MCS and calorimetric estimates result in good agreement, as shown in Figure 2; the average resolution of the MCS estimates on the stopping muon sample is about $15 \%$. A small $(\sim 15 \%)$ underestimation of the MCS measurement w.r.t. calorimetry, appearing for $p \gtrsim 3 \mathrm{GeV} / \mathrm{c}$, is related to the non-perfect planarity of the TPC cathode. This distortion was directly and independently measured after the end of the LNGS run, showing deviations that extend to $\sim 3 \mathrm{~cm}$ (see Figure 3 ). They generate a non-uniformity of the electric field, resulting in spurious track deflections that mimic the effect of MCS. A full 3D field simulation showed that this effect can result in a momentum underestimation of $\sim 10 \%$, largely accounting for the observed underestimation.

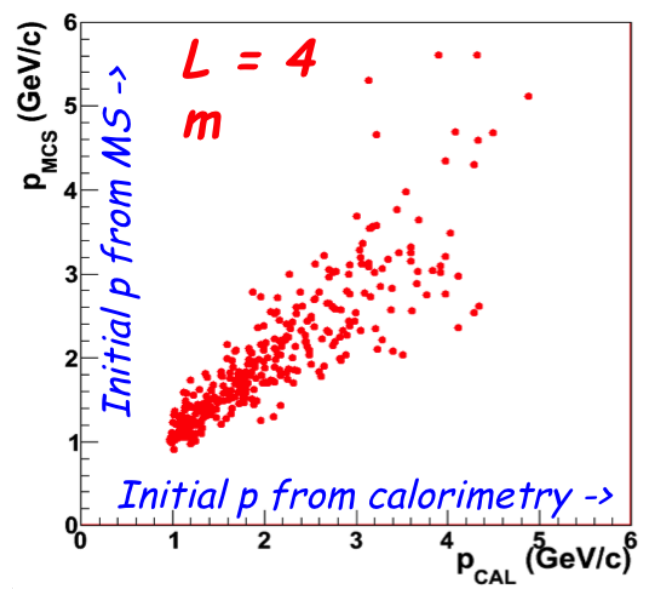

Figure 2. Momentum measured from MCS compared with momentum estimated by calorimetry, for the CNGS stopping muon sample. Muons with track lengths larger than 5 meters were considered, and only the first 4 meter of the muon tracks were used for MCS measurement.

\subsection{Electron/photon separation}

Since electron neutrino interactions usually represent the main signal in many present and future neutrino oscillation experiments, an efficient identification of electrons is a crucial feature of neutrino detectors; in particular, it is very important to efficiently reject the main background given by neutral current interactions with $\pi^{0}$ production.

A unique feature of LAr-TPCs is the very efficient electron-photon separation capability, due to the good spatial and calorimetric resolution; in particular, the accurate measurement of the ionization density $\mathrm{dE} / \mathrm{dx}$ allows to discriminate showers initiated by electrons (characterizing genuine $v_{e}$ interactions) from ones initiated by photons, as illustrated in Figures 4 and 5. 


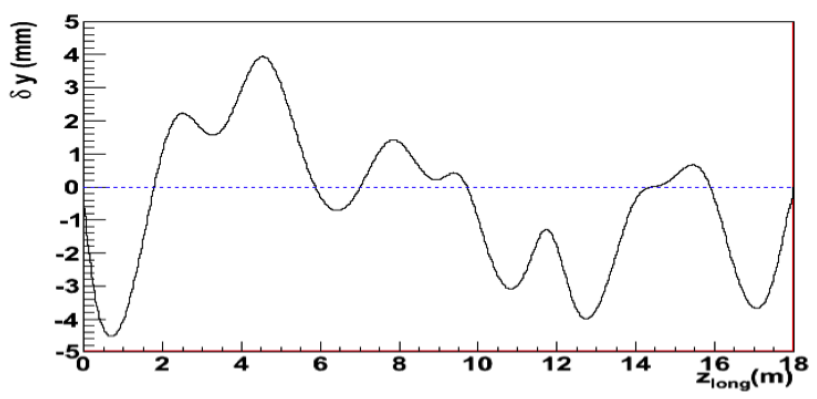

Figure 3. Deviations $\delta y$ from cathode planarity as a function of the longitudinal coordinate $z$, at a height of $75 \mathrm{~cm}$ and a distance of $30 \mathrm{~cm}$ from the cathode plane. The $\delta \mathrm{y}$ values have been interpolated from direct measurements with a laser-meter, performed during the T600 refurbishing at CERN.

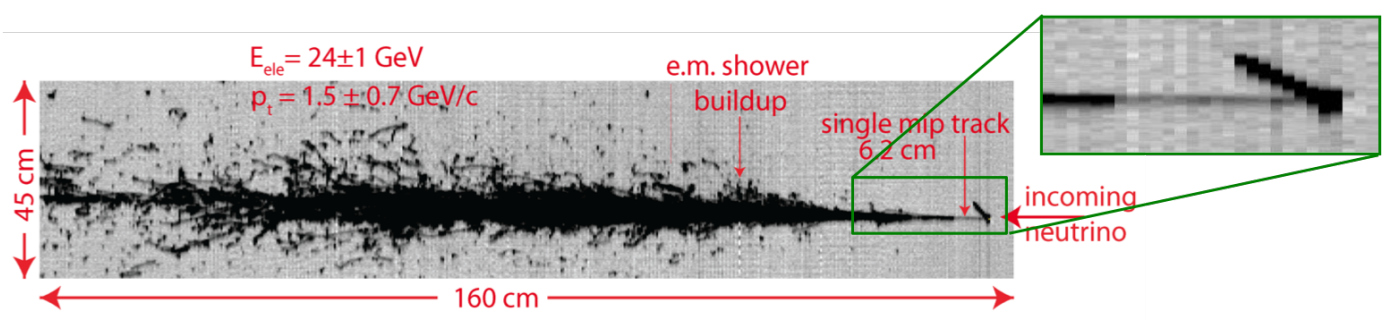

Figure 4. Example of an identified CNGS $v_{e}$ charged current interaction in ICARUS-T600 at LNGS.

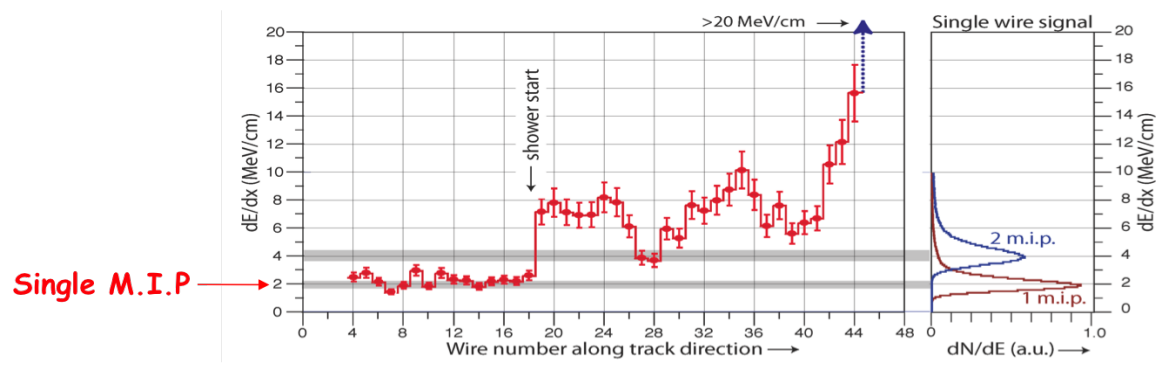

Figure 5. Ionization density as a function of wire number (along the track direction) for the first wires of the event in Figure 4. The grey bands represents $\mathrm{dE} / \mathrm{dx}$ values corresponding to a single m.i.p. $(\sim 2.1 \mathrm{MeV} / \mathrm{cm})$ and a double m.i.p.

\section{Search for sterile neutrinos at the CNGS beam}

ICARUS-T600 searched for possible oscillations mediated by a sterile neutrino in the range identified by LSND $\left(\sim 1 e V^{2}\right)$, by looking for a $v_{e}$ eccess in the CNGS $v_{\mu}$ beam. A sample of $\sim 7.9 \cdot 10^{19}$ pot, corresponding to $\sim 2650$ neutrinos, was considered; the analysis was limited to the energy range between 10 and $30 \mathrm{GeV}$. In this sample, 7 electron neutrino events were found, compared to an expected background of $8.5 \pm 1.1$, dominated by the intrinsical $v_{e}$ beam contamination; the $v_{e}$ identification 
efficiency was estimated from a detailed MonteCarlo study, resulting $\sim 74 \%$ with a negligible contamination from neutral current backgrounds. No excess was therefore observed, allowing to strongly constrain the allowed region for sterile neutrino oscillation in the $\left(\sin ^{2} 2 \theta, \Delta m^{2}\right)$ parameter space, identifying a small area $\left(\sin ^{2} 2 \theta, \Delta m^{2}\right) \sim\left(0.005,0.5 \mathrm{eV}^{2}\right)$ where all available experimental data can be accommodated at $90 \%$ confidence level [4], as summarized in figure 6 .

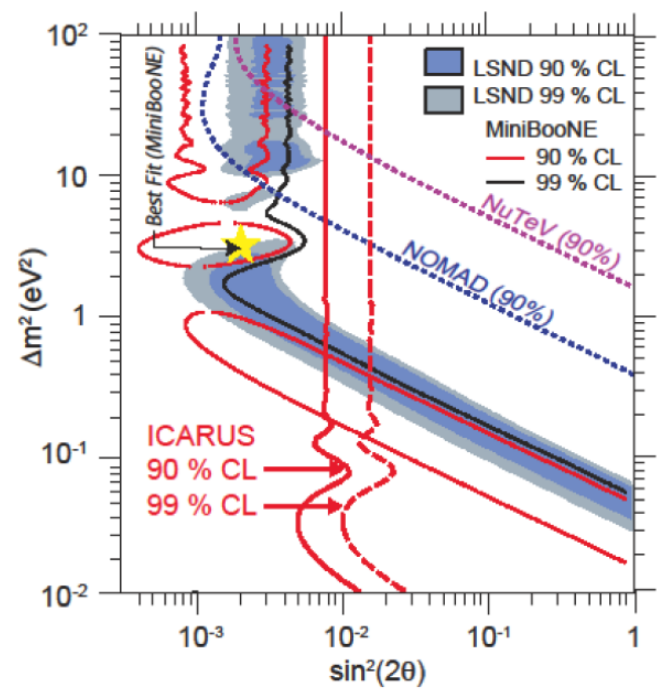

Figure 6. Limits in the sterile neutrino parameter space obtained by the ICARUS-T600 $v_{\mu} \rightarrow v_{e}$ appearance search, compared with LSND and MiniBoone allowed regions and limits from previous experiments.

\section{The SBN experiment}

The phenomenological scenario of sterile neutrino results still shows tension between different experimental results, and requires a solid verification of the flavour change "hints" provided by several experiments. In this complex scenario, the SBN experiment has the goal to clarify this issue by performing both appearance and disappearance measurements with the same beam and detectors.

In this experiment, neutrinos from the Booster beam at FNAL, with an average energy of $\sim 800$ $\mathrm{MeV}$, will be detected by three different LAr-TPC detectors (SBND, MicroBoone and ICARUS-T600) at different distances from the target (about 100, 470 and $600 \mathrm{~m}$ respectively). This approach will allow a greater oscillation sensitivity, based on comparison of spectra at the near and far detector; in absence of anomalous signals due to sterile neutrinos, signals in these detectors should be identical, except for an obvious solid-angle factor, as illustrated in Figure 7. Moreover, the use of the same technology for all detectors will allow a strong reduction of systematics related to $v$-Ar cross sections and to event identification and selection.

The appearance measurement will allow to fully cover the currently allowed region in the parameter space with $99 \%$ confidence level in 3 years of data taking, corresponding to $6.6 \cdot 10^{20}$ pot, as shown in Figure 8. On the other hand, no positive sterile neutrino signal has been recorded so far in the $v_{\mu}$ disappearance channel; the SBN experiment will be able to extend the current limits by about one order of magnitude. The appearance and disappearance measurements will be dependent on different 

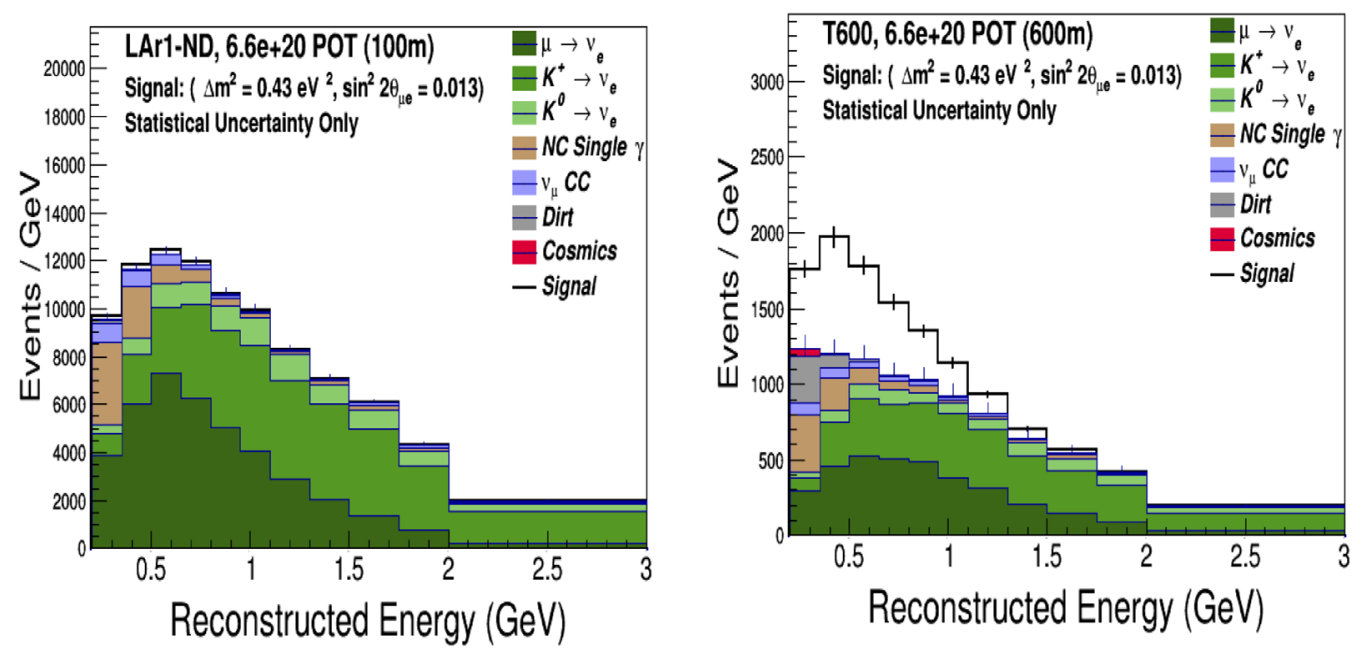

Figure 7. Energy spectrum of all $v_{e}$ events expected at SBND near detector (left) and ICARUS-T600 far detector (right) for 3 years of data taking at the Booster beam. The expected signal for a reference sterile neutrino case is shown in white, while the various background components are in colors.

mixing angles, parametrizing the mixing of the active flavors to the hypothetical sterile neutrino; this will allow to test both these mixings with the same experiment.

\section{ICARUS-T600 overhauling at CERN}

The ICARUS-T600 detector is currently undergoing a massive overhauling at CERN, in view of its use for the SBN experiment. This program (WA104) will introduce substantial improvements while keeping the present detector performance. In particular, the refurbishing work will involve several detector subsystems:

- New cold vessels, with a purely passive insulation;

- Renovated cryogenics and purification equipment;

- Reduction of the cathode non-planarity discussed in section 2.2 to a few millimeters;

- Upgrade of the light collection system, in order to achieve a high timing resolution (few ns), needed for the precise localization of events within the drift window; this is required for an efficient rejection of cosmic background. The number of PMTs will also be increased to 360, corresponding to $\sim 5 \%$ coverage.

- New faster, higher-performance read-out electronics. In particular, the synchronous sampling on the whole detector will allow to improve MCS momentum resolution to $\sim 12 \%$.

The T600 detector is scheduled to be transported to FNAL during 2017, to start installation and commissioning. 


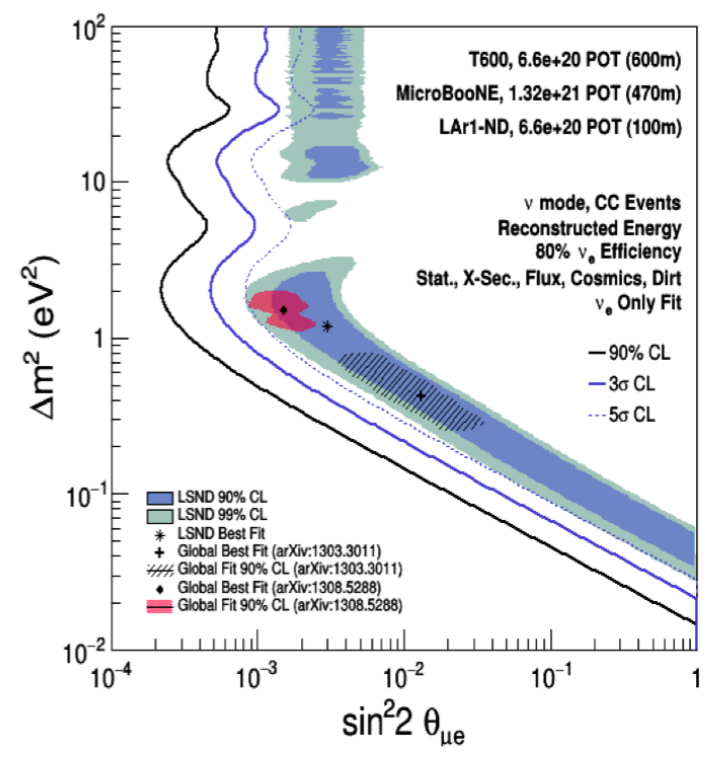

Figure 8. Expected sensitivity of the SBN program $v_{\mu} \rightarrow v_{e}$ appearance search for 3 years of data taking, compared with the LSND allowed region and two different global fits to all experimental data (see figure for references).

\section{Conclusions}

The successful ICARUS-T600 run at LNGS proved the maturity of the LAr-TPC technology, and represents a milestone towards future larger-scale detectors. It also provided interesting physics results, including strong constraints on sterile neutrinos.

Nevertheless, the sterile neutrino puzzle remains open and further experimental tests of this hypothesis are needed. The SBN experiment will definitely clarify the situation, by performing highstatistics, accurate searches in both the appearance $\left(v_{\mu} \rightarrow v_{e}\right)$ and disappearance $\left(v_{\mu} \rightarrow v_{\mu}\right)$ channels.

\section{References}

[1] S. Amerio et al. (ICARUS Coll.), NIM A527, 329 (2004)

[2] C. Rubbia et al. (ICARUS Coll.), JINST 6, P07011 (2011)

[3] M. Antonello et al. (ICARUS Coll.), JINST 9, P12006 (2014)

[4] M. Antonello et al. (ICARUS Coll.), Eur.Phys.J C73, 2599 (2013)

[5] R. Acciarri et al., A Proposal for a Three Detector Short-Baseline Neutrino Oscillation Program in the Fermilab Booster Neutrino Beam, arXiv:1503.01520 